\title{
A genetic algorithm to calibrate dynamical systems: Confidence intervals for parameters and residuals
}

\author{
Antonio Caselles \\ IASCYS member \\ Departament de Matemàtica Aplicada \\ Universitat de València \\ Valencia, Spain \\ Antonio.Caselles@uv.es
}

\author{
Joan C. Micó \\ Intitut Universitari de Matemàtica Multidisciplinar \\ Universitat Politècnica de València \\ Valencia, Spain \\ jmico@mat.upv.es
}

\author{
Salvador Amigó. \\ Departament de Personalitat, Avaluació i Tractaments Psicològics. \\ Universitat de València \\ Valencia, Spain \\ Salvador.Amigo@uv.es
}

\begin{abstract}
This paper presents a genetic algorithm to calibrate dynamical systems that is able to calculate confidence intervals for the parameters of the system. As an application case is used to calibrate the system that reproduces the dynamical response of the General Factor of Personality (GFP) to a given stimulus, particularly to a stimulant drug dose. The model is called in Literature as the response model and includes an integro-differential equation. The presented application case is a single case ABC experimental design where the stimulus is methylphenidate.
\end{abstract}

Keywords-General Factor of Personality; genetic algorithm; dynamics; integro-differential equation; calibration; methylphenidate.

\section{Introduction}

The here presented genetic algorithm has been designed for a particular case but, changing the corresponding system equations it can be used to calibrate any other system. The considered particular case is a system that determines the evolution of the personality of a subject as a consequence of receiving a certain stimulus, for instance a drug dose. In the application case here presented, methylphenidate is the drug being used. It is a powerful psycho-stimulant. This psycho-stimulation can be measured by the General Factor of Personality (GFP), as a universal observable feature of personality. A questionnaire containing the five adjectives scale that is described by Amigó, Micó \&
Caselles [3][5] and constructed specifically to assess GFP in the context of the Unique Trait Personality Theory (UTPT) [1][5] is used for the considered case. The UTPT claims for a unique trait, as synonymous of single trait, substituted later by the equivalent concept of GFP, to represent the overall human personality. The GFP is the psychological expression of the activation level of the organism stress system. In fact, in the context of the UTPT, GFP is called also extraversion in a wider sense than the one used in behavioral science, i.e., in the sense of activation level of the organism stress system.

The evolution of the GFP is calculated by the response model that is an integro-differential equation that has been widely assessed in the context 
of different experimental designs. It can reproduce the acute effect of a stimulant drug [2][6][9][10][11]. The model reproduces the dynamical pattern forecasted by Solomon \& Corbit [13] and Grossberg [7], by using the hedonic scale, and by Amigó [1] for the GFP, i.e., a typical inverted-U.

The here performed calibration of the model is based on a genetic algorithm. Genetic algorithms (GAs) are Evolutionary Algorithms (EAs) (they adapt their parameters according to previous results) that try to imitate Natural Selection inside a population through parent selection, recombination, mutation and migration. About details on GAs and its use in systems calibration, see for instance: Whitley, [14], Guzmán-Cruz et al., [8] and Muraro \& Dilao [12]. Nevertheless there are a lot of possible options for their definition, obviously related on how to perform selection, crossover and mutation. The here introduction of immigration could be a novelty.

\section{The response model}

The kinetic part of the response model provides the evolution of the stimulus amount $s(t)$, present in plasma after intake by the individual. It is given by the time function:

$$
\left\{\begin{array}{r}
s(t)= \\
\frac{\alpha \cdot M}{\beta-\alpha}(\exp (-\alpha \cdot t)-\exp (-\beta \cdot t)): \alpha \neq \beta \\
\alpha \cdot M \cdot t \cdot \exp (-\alpha \cdot t): \alpha=\beta
\end{array}\right.
$$

Equation (1) is the solution of two coupled differential equations [11], which assumes that no drug/stimulus is present in the organism before consumption. In (1) $M$ is the initial amount of a drug single dose, $\alpha$ is the stimulus assimilation rate and $\beta$ is the stimulus elimination rate. The dynamics of the GFP is given by the following integro-differential equation [11]:

$$
\left.\begin{array}{c}
\frac{d y(t)}{d t}=a(b-y(t))+\frac{p}{b} s(t) \\
-b \cdot q \cdot \int_{0}^{t} \mathrm{e}^{\frac{x-t}{\tau}} \cdot s(x) \cdot y(x) d x \\
y(0)=y_{0}
\end{array}\right\}
$$

In (2), $s(t)$ represents the stimulus; $y(t)$ represents the GFP dynamics; and $b$ and $y_{0}$ are respectively its tonic level and its initial value. Its dynamics is a balance of three terms, which provide the time derivative of the GFP: the homeostatic control $a(b-$ $y(t))$, i.e., the cause of the fast recovering of the tonic level $b$, the excitation effect $p \cdot s(t) / b$, which tends to increase the GFP, and the inhibitor effect $\int_{0}^{t} \mathrm{e}^{\frac{x-t}{\tau}}$. $s(x) \cdot y(x) d x$, which tends to decrease the GFP and is the cause of a continuously delayed recovering, with the weight $\mathrm{e}^{\frac{x-t}{\tau}}$. Parameters $a, p, q$ and $\tau$ are named respectively the homeostatic control power, the excitation effect power, the inhibitor effect power and the inhibitor effect delay. All the parameters of the model depend on the individual personality or individual biology and on the type of stimulus.

\section{The genetic algorithm used for the response model calibration}

The program we use for calibration has been ad hoc designed for the previously described model but it can be adapted easily for systems with the following characteristics:

(1) Real data are deterministic. In the case of the response model, real GFP is measured by the responses of an individual to a questionnaire every some minutes. And model parameters are specific of the individual.

(2) The system to be calibrated is deterministic.

(3) All parameters have a continuous range of possible or plausible values from a maximum to a minimum value.

(4) A single objective variable (function) must be considered, but it may be designed as a weighted combination of several other ones.

(5) Parameter space (search space) is a multidimensional compact space (continuity is assumed in parameter values inside a range of possible or plausible values).

(6) In order to assure the global character of the found optimum three strategies are considered:

a. A random sample may be analyzed, from the entire search space or from specific zones, in order to identify starting points.

b. Random migrants with reproduction capacity are introduced inside the current population in every generation.

c. Several iterations are performed using the previous optimum as a new starting point, up to no improvement is found or the top number of iterations is reached. 


\subsection{The needed data}

The response mode has seven parameters: $\alpha, \beta, \mathrm{a}, \mathrm{b}$, $\mathrm{p}, \mathrm{q}, \tau$ and $\mathrm{M}$ (M may also be adjusted like the other parameters when the stimulus is not measurable, for instance: a placebo), which meaning has been previously explained. A vector of nine components containing a value for each parameter plus the corresponding GFP (y) may be considered as an individual of a population of possible characterizations of the system. The staring values of the parameters (given by previous knowledge), their maximum values, their minimum values, their search window width (\% of their initial value), and their search step width (\% of their initial value) have to be introduced at the beginning of the search process. Other needed data are the number of experimental values, their time step, and their values. The integration method (Euler or Runge-Kutta-4) and the integration step size have to be also specified. The function to be optimized may be the mean squared deviation $\left(s^{2}\right)$, the determination coefficient $\left(\mathrm{R}^{2}\right)$ or the relative mean deviation.

The GA may be optionally used, and in the case it is used the following options must be specified: number of individuals of the population, percentage of the population corresponding to reproducers (the best individuals), number of immigrants per generation, mutant genes per thousand in a new individual, number of generations inside a given iteration, and maximum number of iterations. In the case of not using the GA but only analyzing a sample, it may be exhaustive or uniformly random. This sampling process also admits iterations.

\subsection{The GA pseudo-code}

The proposed GA intends to be the simplest possible one in order to be as fast as possible without restricting the possibility to find a global optimum. The following pseudo-code might be enough descriptive of the here presented $G A$ that we name PARDOSU.

Introduce data and options

Define the initial population (vectors with random values for parameters and the objective function value)
For $\mathrm{i}=1$ to "number of iterations", do:

For $\mathrm{j}=1$ to "number of generations", do:

Arrange population from lower to higher the objective function

Retain the best individuals and eliminate the remaining ones

Incorporate some immigrants (randomly defined inside parameters' ranges)

"Complete the population by reproduction (with mutation) of the present individuals, i.e.:"

For k="number of reproducers" +1 to "population size", do:

Choose randomly the "father" and the "mother" of the new individual

For each gen (parameter) choose randomly whether it comes from "father" or "mother"

For each gen (parameter) choose randomly whether it is newly randomly defined or not

Next new individual

Next generation

If "previous optimum is not improved" Then ExitIterations-Loop Else Continue

Use the optimum individual as new starting point

Next iteration

Calculate residuals by comparing the found optimum with the experimental values

Test residuals for Normality and zero-mean

If "yes" Then "calculate confidence intervals to define the optimal fitting evolution band"

For $\mathrm{j}=1$ to "number of parameters + objective function" do:

Test parameter $\mathrm{j}$ for normality inside the best individuals group

If p-value for normality is acceptable Then

Calculate and write the corresponding mean, standard deviation, chi-squared, $\mathrm{t}$ of Student and the upper and lower bounds of the confidence interval

Else write only the corresponding mean, standard deviation and chi-squared

End If

Next parameter

Write all other results

\section{The response model calibration}

The studied application case consists in one subject that consumed $20 \mathrm{mg}$ of methylphenidate. The Five Adjectives scale questionnaire (adventurous, daring, enthusiastic, merry and bored) was filled out before consumption and after consumption every 15 
minutes during 4 hours. The interval of the GFP measures is $y \in[0,25]$. The calibration result of the response model for the GFP dynamics is provided in Figure 1. With respect to parameters, inside the best individuals group, most of them were not normally distributed (very high chi-squared values), others were constants or with relatively low standard deviations. Note in Figure 1 that it considers the confidence intervals, for a $95 \%$ of confidence level, provided by the random variablility values of the parameters.

\section{Conclusions}

Figure 1 shows the calibration result of the response model for the GFP response as a consequence of 20 mg of methylphenidate obtained with the proposed $G A$. The obtained determination coefficient value $\mathrm{R}^{2}$ supports model applicability as in other studies from literature (see Section 1). The algorithm shows a good performance and time efficiency.

For future work we aim to compare the efficiency of the present features of PARDOSU with alternative specific features, such as for instance: mutation of each parameter restricted to values close to the present one, optional equipotency of gens (at present all gens are dominant/recessive), and incest prevention. Options such as selection by competition are discarded due to they do not guarantee the permanence of the best individuals inside the population.

\section{REFERENCES}

[1] Amigó, S. (2005). La teoría del rasgo único de personalidad. Hacia una teoría unificada del cerebro y la conducta (The Unique Personality Trait Theory. Towards a unified theory of brain and behavior). Editorial de la Universitat Politècnica de València. Valencia, Spain.

[2] Amigó, S., Caselles, A. \& Micó, J.C. (2008). A dynamic extraversion model: the brain's response to a single dose of a stimulant drug. British Journal of Mathematical and Statistical Psychology, 61, 211231.

[3] Amigó, S., Micó, J.C. \& Caselles, A. (2009a). Five adjectives to explain the whole personality: a brief scale of personality. Revista Internacional de Sistemas, 16, 41-43. (Available in http://www.uv.es/caselles/ )

[4] Amigó, S., Caselles, A., Micó, J.C. \& García, J.M. (2009b). Dynamics of the unique trait of personality: blood's glutamate in response to methylphenidate and conditioning. Revista Internacional de sistemas, 16, 35-40.

[5] Amigó, S., Caselles, A. \& Micó, J.C. (2010). The General Factor of Personality Questionnaire (GFPQ): Only one factor to understand the personality? The Spanish Journal of Psychology, 13, 5-17.

[6] Caselles, A., Micó, J.C., Amigó, S. 2011. Dynamics of the General Factor of Personality in response to a single dose of caffeine. The Spanish Journal of Psychology, 14, 675-692.

[7] Grossberg, S. (2000). The imbalanced DBRAIN: from normal to schizophrenia. Biological Psychiatry, 48, 81-98.

[8] Guzmán-Cruz, R., Castañeda-Miranda, R., García-Escalante, J.J., López-Cruz, J.L. (2009). Calibration of a greenhouse climate model using evolutionary algorithms. Biosystems Engineering, 104, 135-142.

[9] Micó, J.C., Amigó S. \& Caselles, A. (2012). Changing the General Factor of Personality and the c-fos expression with methylphenidate and SelfRegulation Therapy. The Spanish Journal of Psychology, 15(2): 850-867.

[10] Micó, J.C., Caselles, A., Amigó, S., Cotolí, A. \& Sanz, M.T. (2013). A mathematical approach to the body-mind problem from a System Personality theory. Systems Research and Behavioral Science, 30, 735-749.

[11] Micó, J.C., Amigó, S. \& Caselles, A. (2014). From the Big Five to the General Factor of Personality: a Dynamic Approach. The Spanish Journal of Psychology, 17, e74, 1-18.

[12] Muraro, D., Dilao,R., (2013). A parallel multiobjective optimization algorithm for calibration of mathematical models. Swarm and Evolutionary computation, 8, 13-25.

[13] Solomon, R.L. \& Corbit, J.D. (1974). An opponent-process theory of motivation. I. Temporal 
dynamics of affect. Psychological Review, 81, 119145.

[14] Whitley, D., (2001) . An overview of evolutionary algorithms: practical issues and common pitfalls. Information and software technology, 43, 817-831.

[1] Amigó, S. (2005). La teoría del rasgo único de personalidad. Hacia una teoría unificada del cerebro y la conducta (The Unique Personality Trait Theory. Towards a unified theory of brain and behavior). Editorial de la Universitat Politècnica de València. Valencia, Spain.

[2] Amigó, S., Caselles, A. \& Micó, J.C. (2008). A dynamic extraversion model: the brain's response to a single dose of a stimulant drug. British Journal of Mathematical and Statistical Psychology, 61, 211231.

[3] Amigó, S., Micó, J.C. \& Caselles, A. (2009a). Five adjectives to explain the whole personality: a brief scale of personality. Revista Internacional de Sistemas, 16, 41-43. (Available in http://www.uv.es/caselles/ )

[4] Amigó, S., Caselles, A., Micó, J.C. \& García, J.M. (2009b). Dynamics of the unique trait of personality: blood's glutamate in response to methylphenidate and conditioning. Revista Internacional de sistemas, 16, 35-40.

[5] Amigó, S., Caselles, A. \& Micó, J.C. (2010). The General Factor of Personality Questionnaire (GFPQ): Only one factor to understand the personality? The Spanish Journal of Psychology, 13, 5-17.

[6] Caselles, A., Micó, J.C., Amigó, S. 2011. Dynamics of the General Factor of Personality in response to a single dose of caffeine. The Spanish Journal of Psychology, 14, 675-692.

[7] Grossberg, S. (2000). The imbalanced DBRAIN: from normal to schizophrenia. Biological Psychiatry, 48, 81-98.

[8] Guzmán-Cruz, R., Castañeda-Miranda, R., García-Escalante, J.J., López-Cruz, J.L. (2009). Calibration of a greenhouse climate model using evolutionary algorithms. Biosystems Engineering,
104, 135-142.

[9] Micó, J.C., Amigó S. \& Caselles, A. (2012). Changing the General Factor of Personality and the c-fos expression with methylphenidate and SelfRegulation Therapy. The Spanish Journal of Psychology, 15(2): 850-867.

[10] Micó, J.C., Caselles, A., Amigó, S., Cotolí, A. \& Sanz, M.T. (2013). A mathematical approach to the body-mind problem from a System Personality theory. Systems Research and Behavioral Science, 30, 735-749.

[11] Micó, J.C., Amigó, S. \& Caselles, A. (2014). From the Big Five to the General Factor of Personality: a Dynamic Approach. The Spanish Journal of Psychology, 17, e74, 1-18.

[12] Muraro, D., Dilao,R., (2013). A parallel multiobjective optimization algorithm for calibration of mathematical models. Swarm and Evolutionary computation, 8, 13-25.

[13] Solomon, R.L. \& Corbit, J.D. (1974). An opponent-process theory of motivation. I. Temporal dynamics of affect. Psychological Review, 81, 119145.

[14] Whitley, D., (2001) · An overview of evolutionary algorithms: practical issues and common pitfalls. Information and software technology, 43, 817-831.

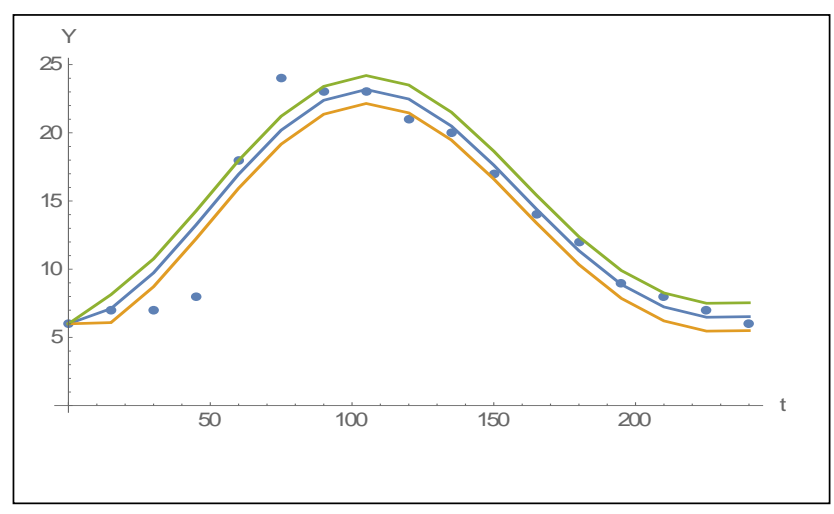

Figure 1: GFP (Y) versus time $(\mathrm{t})$. Experimental values (dots) and the calibrated response model (line). $\mathrm{R}^{2}=0.92$. 